\title{
Graph Neural Network based on Geometric and Appearance Attention for 6D Pose Estimation
}

\author{
Tianfu wang \\ State Key Laboratory of Robotics, Shenyang Institute of \\ Automation, Chinese Academy of Sciences, Shenyang \\ 110016, China; Institutes for Robotics and Intelligent \\ Manufacturing, Chinese Academy of Sciences, Shenyang \\ 110169, China; University of Chinese Academy of Sciences, \\ Beijing 100049, China \\ wangtianfu@sia.cn
}

\begin{abstract}
Object 6D pose estimation from RGB-D images requires extracting useful information from two complementary data sources (color and depth). Usually, previous methods extract from two networks (for color and depth) separately and concatenate the final features. In this work, we propose a novel attention based graph neural network to process color and depth images concurrently. In this way, we can learn the interactions between depth and color, leading to an effective fusion. Specifically, our method consists of two stages. First, we convert the segmentation of an RGB-D image into colored point clouds. Second, we estimate the poses of objects by an attention based graph neural network. Our attention mechanism is based on geometric and appearance information. In this way, 3D geometric and 2D appearance information can be fully utilized for better feature learning. The experimental results on LineMOD and Occlusion LineMOD datasets show the effectiveness of our method.
\end{abstract}

\section{CCS CONCEPTS}

- Computing methodologies; • Artificial intelligence; • Computer vision; • Vision for robotics; • Machine learning;

\section{KEYWORDS}

6D pose estimation, Graph neural network, Colored point clouds

\section{ACM Reference Format:}

Tianfu wang and Hongguang Wang. 2021. Graph Neural Network based on Geometric and Appearance Attention for 6D Pose Estimation. In 2021 4th International Conference on Artificial Intelligence and Pattern Recognition (AIPR 2021), September 24-26, 2021, Xiamen, China. ACM, New York, NY, USA, 6 pages. https://doi.org/10.1145/3488933.3488959

\section{INTRODUCTION}

$6 \mathrm{D}$ object pose estimation is typically defined as estimating the $3 \mathrm{D}$ orientation (rotation) and translation of the object. Knowing the pose of an object is important for many real-world applications.

Permission to make digital or hard copies of all or part of this work for personal or classroom use is granted without fee provided that copies are not made or distributed for profit or commercial advantage and that copies bear this notice and the full citation on the first page. Copyrights for components of this work owned by others than ACM must be honored. Abstracting with credit is permitted. To copy otherwise, or republish, to post on servers or to redistribute to lists, requires prior specific permission and/or a fee. Request permissions from permissions@acm.org.

AIPR 2021, September 24-26, 2021, Xiamen, China

(C) 2021 Association for Computing Machinery.

ACM ISBN 978-1-4503-8408-7/21/09..\$15.00

https://doi.org/10.1145/3488933.3488959

\author{
Hongguang Wang \\ State Key Laboratory of Robotics, Shenyang Institute of \\ Automation, Chinese Academy of Sciences, Shenyang \\ 110016, China; Institutes for Robotics and Intelligent \\ Manufacturing, Chinese Academy of Sciences, Shenyang \\ 110169, China \\ hgwang@sia.cn
}

For example, a robot must be able to obtain the pose of the target object to manipulate the object. Due to the lack of geometric information, the RGB-only method is less effective in estimating the poses of low-textured objects. The advent of low price RGB-D sensors provides geometric information to solve this problem and raises a new question: How can we extract powerful features from two data sources (color and depth) for accurate pose estimation?

Classical methods first extract features from RGB-D images and then estimate the object poses through the correspondence groups $[2,4,5]$. However, these methods are limited by hand-crafted features and not learnable to fit the data. Recently, with the dramatic growth of deep learning, the data-driven method becomes more popular. There are mainly two lines of works. In the first line, these methods first estimate an initial pose from color image and then refine it on point clouds using an Iterative Closest Point (ICP) [1] or a multi-view hypothesis verification, such as PoseCNN [23] and Multi-Class pose Network (MCN) [9]. These refinement steps are time-consuming and cannot be optimized with the final goal. The other line of works, such as DenseFusion [22], extract features from RGB-D data with heterogeneous architecture. Despite being effective, the two networks are not able to exchange or share information during the feature learning stage.

In this paper, we convert the RGB-D data into colored point clouds to make a single data format containing geometric and appearance information. This allows us to extract geometric and appearance features with a single network architecture. However, the network architecture which is used in previous work [22] cannot extract powerful features from this data format. To this end, we propose a novel attention based graph neural network for 6D pose estimation. The proposed graph neural network extracts features with geometric and appearance information from colored point clouds. In each stage of feature learning, geometric and appearance information can be exchanged and shared with each other. In addition, in order to make our network extract more powerful features, we design an attention mechanism based on geometric and appearance information. Furthermore, thanks to our colored point clouds data format, we use colored ICP [16] for refinement in hard cases, which is more robust and more accurate than ICP.

Our contributions can be summarized as: 1) We propose a novel graph neural network for $6 \mathrm{D}$ pose estimation from colored point clouds. 2) We design a novel attention based graph neural network. In addition, the attention mechanism is based on geometric and appearance information. 


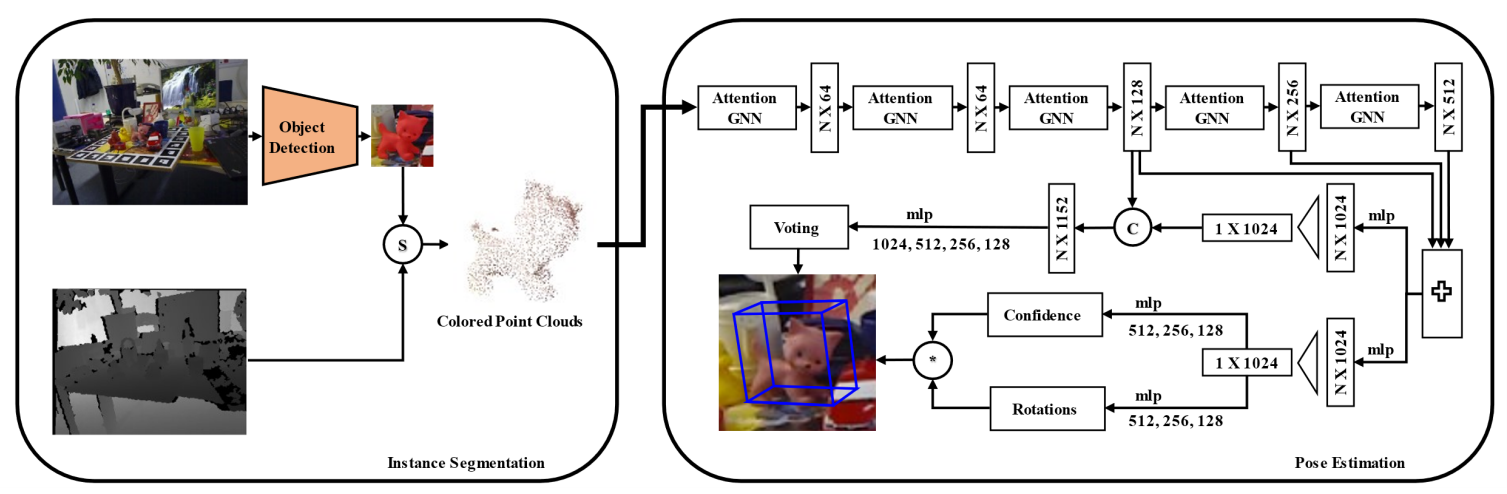

Figure 1: Overview of our method. Given an RGB-D image, we first generate the object mask by a semantic segmentation network, and then we extract colored point clouds from masked color pixel and depth pixel. Appearance and geometric features are obtained through attention based GNN, from which rotation and translation of object are predicted in two separate branches.

\section{RELATED WORK}

\subsection{Pose Estimation with RGB Data}

Traditional methods are based on keypoint [13] of known object models or template matching [5]. The 6D poses are estimated by solving the PnP problem. Newer methods use a deep learning framework to estimate the poses of objects, which can be divided into direct methods and indirect methods. Direct methods [3, 7, 8, 14] directly output estimated poses from an RGB image. Though it has advantages in running speed, these methods perform poorly because of the nonlinearity of the rotating space. Instead, indirect methods $[6,18,20]$ establish 2D-3D correspondences between 2D keypoints and 3D keypoints, then solve poses by PnP. However, the lack of geometric information limits the performance of RGB-based methods.

\subsection{Pose Estimation with RGB-D Data}

Depth images can provide geometric information for texture-less objects. Classic methods $[4,5,19]$ utilize hand-designed features or templates from RGB-D data and obtain the pose through the correspondence. Recent data-based methods [23] use a neural network to estimate the initial poses from the RGB image and refine them with point clouds using ICP [1]. However, the process of iterative refinement is very time-consuming and difficult to apply in realtime applications. Instead, $[15,21,22]$ predict $6 \mathrm{D}$ poses from RGB-D images in an end-to-end way. [15] concatenate the depth image as the fourth channel to the RGB image. Nevertheless, this method neglects the intrinsic 3D structure of the depth image. [22] convert depth image to point clouds. They fuse the features extracted by two different networks. [21] proposes discrete-continuous formulation for rotation prediction on this basis, and uses RANSAC-based voting to predict translation. The network in $[15,21,22]$ extracts geometric and appearance information separately, the two networks are not able to exchange or share information in the feature learning stage, which limits the ability of feature learning. In this work, we use colored point clouds and a graph neural networks to extract features. Specifically, we use the attention mechanism based on geometry and appearance to make full use of two streams of information.

\section{METHODOLOGY}

In this work, we aim at estimating $6 \mathrm{D}$ poses of a set of known objects from an RGB-D image of a cluttered scene. $6 \mathrm{D}$ pose of the object estimated from the image is a rigid transformation from the object coordinate system to the camera coordinate system. This rigid transformation is composed of a translation $t \in \mathbb{R}^{3}$ and a rotation $R \in S O(3)$.

\subsection{Overview}

Figure 1 illustrates the framework of our approach for 6D pose estimation. Our approach consists of two main steps: instance segmentation and pose estimation. The first step is to detect the objects of interest in the color image and obtain a foreground mask. We use a well-developed segmentation architecture proposed by [23]. Then we extract colored point clouds from the masked color pixel and depth pixel.

The second stage processes the colored point clouds and estimates the object's $6 \mathrm{D}$ pose. An attention based graph neural network is used to extract geometric and appearance features from colored point clouds. Then we predict rotation and translation in two separate branches. Concretely, the rotation is solved by a robust discrete-continuous regression scheme, while the translation is by voting to the center.

\subsection{Attention based Graph Neural Network}

Given an aligned RGB-D image, we first convert them to colored point clouds $P=\left\{\left[p_{1}, a_{1}\right],\left[p_{1}, a_{2}\right], \ldots,\left[p_{N}, a_{N}\right]\right\} \in \mathbb{R}^{6}$, where $p_{i}=[x, y, z]$ is the position and $a_{i}=\{r, g, b\}$ is the image pixel value. We compute a directed graph $\mathcal{G}=(\mathcal{V}, \mathcal{E})$ from a given colored point cloud $P$ according to their spatial neighbors, where $\mathcal{V}=\{1,2, \ldots, N\}$ and $\mathcal{E} \subseteq|\mathcal{V}| \times|\mathcal{V}|$ are the vertices and edges. In the simple case, we construct $\mathcal{G}$ as the k-nearest neighbor (K$\mathrm{NN})$ graph of $P$ in geometric space. The self-loop is added in the graph, meaning each vertice has an edge with itself. Let $\mathcal{N}(i)=$ 


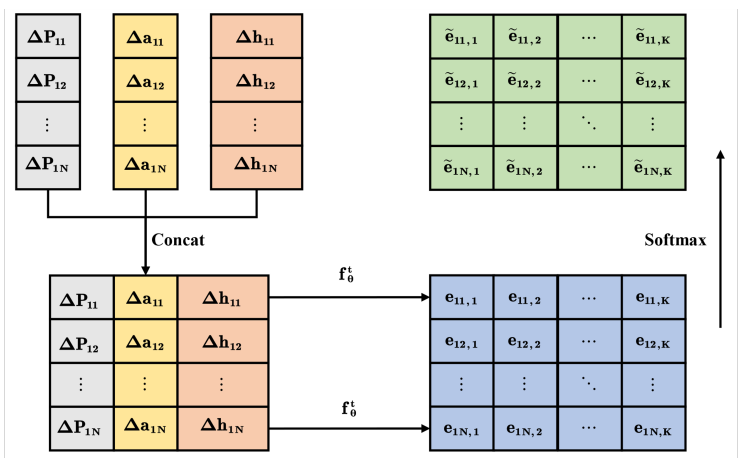

Figure 2: The attention mechanism. It receives relative geometry, appearance, and feature as input and then normalizes them to attentional weights.

$\{j:(i, j) \in E\} \cup\{i\}$ be the neighbor set of the vertex $i$. Denote $H=\left\{h_{1}, h_{2}, \ldots, h_{N}\right\}$ as the set of input features.

A typical graph neural network extract features by aggregate vertices along the edges. In the $(t+1)^{t h}$ iteration, it updates each vertex feature as follow:

$$
\begin{gathered}
h_{i}^{t+1}=g^{t}\left(\rho^{t}\left(\left\{e_{i j}^{t} \mid(i, j) \in E\right\}\right), h_{i}^{t}\right) \\
e_{i j}^{t}=f^{t}\left(h_{i}^{t}, h_{j}^{t}\right)
\end{gathered}
$$

where $e_{i j}^{t}$ is the edge feature. $h_{i}^{t}$ is the vertex feature. $f^{t}($.$) is the$ function that computes the edge feature between vertexes. $\rho^{t}($.) aggregate the edge features. $g^{t}($.$) update vertex with aggregated$ edge features.

The importance of the points on the model point clouds should be different, such as keypoints on the point clouds should be more important. We construct an attention mechanism to make our graph neural network focus on the most important part of the neighbors for feature learning. Specifically, we compute the attentional weights of edges based on geometry, appearance, and feature in the form (as shown in Figure 2):

$$
e_{i j}^{t}=f^{t}\left(p_{j}-p_{i}\left\|a_{j}-a_{i}\right\| h_{j}^{t}-h_{i}^{t}\right)+f_{\phi}^{t}\left(c_{i}\left\|p_{i}\right\| h_{i}^{t}\right)
$$

where $\|$ is the concatenation operation. $f_{\theta}^{t}$ represents multilayer perceptrons. It is worth noting that, we use relative geometry and relative appearance within the neighborhood. The relative information can provide translation invariance and luminosity invariance, which makes our network more robust. To make the coefficients between different vertexes easy to compare, we normalize them across all choices of $j$ using the softmax function:

$$
\tilde{e}_{i j}^{t}=\operatorname{softmax}\left(e_{i j}^{t}\right)=\frac{\exp \left(e_{i j}^{t}\right)}{\sum_{k \in \mathcal{N}_{i}} \exp \left(e_{i k}^{t}\right)}
$$

We model the $g^{t}$ using multilayer perceptrons (MLP). Therefore, the final output of the proposed attention based graph neural network can be formulated as follows:

$$
h_{i}^{t+1}=M L P_{g}^{t}\left(\frac{1}{N} \sum_{j \in \mathcal{N}(i)} \tilde{e}_{i j}^{t} * M L P_{f}^{t}\left(h_{j}^{t}\right)\right)+h_{i}^{t}
$$

Where $*$ represents the element-wise product of two vectors.

\subsection{Pose Estimation}

After extracting the features, we follow [21] to robustly estimate the poses of objects. Specifically, we use MLP to estimate the vector from the point $\mathbf{p}$ to the center point $\mathbf{c}$ from the feature:

$$
\mathbf{v}=\frac{\mathbf{c}-\mathbf{p}}{|\mathbf{c}-\mathbf{p}|_{2}}
$$

where $\mathbf{v}$ is a unit vector from point to the center, and use RANSAC for voting $[18,23]$ to get the coordinate of center.

For rotation, we predict a deviation $\Delta R_{i}$ at rotation anchor $\hat{R}_{i}$ to get the final rotation $R_{i}=\Delta R_{i} \hat{R}_{i} . \Delta R_{i}$ is constrained to be within the vicinity of $\hat{R}_{i}$ :

$$
L_{r e g}=\sum_{i} \max \left(0, \max _{j \neq i}\left\langle\mathbf{q}_{i}, \hat{\mathbf{q}}_{j}\right\rangle-\left\langle\mathbf{q}_{i}, \hat{\mathbf{q}}_{i}\right\rangle\right)
$$

where $\mathrm{q}_{\mathrm{i}}$ and $\hat{\mathrm{q}}_{\mathrm{i}}$ are the quaternion representations of $R_{i}$ and $\hat{R}_{i}$. We predict an uncertainty score $\sigma_{i}$ for each anchor in the icosahedral group [24], which is uniformly sampled from $S O(3)$ :

$$
L_{R}=\sum_{i} \ln \sigma_{i}+\frac{d_{i}}{\sigma_{i}}
$$

where $d_{i}$ is the normalized ShapeMatch-Loss [23] $d_{i}=L_{i} / d, d$ is the object diameter. The minimum $L_{R}$ is achieved when $\sigma_{i}=d_{i}$. During inference, we choose the smallest uncertainty score as the final output.

\section{EXPERIMENTS}

\subsection{Benchmark Datasets}

To validate our method, we evaluate it on two challenging $6 \mathrm{D}$ objects pose estimation datasets: LineMOD dataset [4] and Occlusion LineMOD dataset [2].

LineMOD contains 13 low-textured objects. The dataset contains 13 RGB-D video sequences and is annotated with 6D poses and instance masks. This dataset has the following challenges: cluttered scenes, texture-less objects, and lighting condition change. We fellow previous data-based methods $[10,20,22]$ to split the training and testing set.

Occlusion LineMOD is a subset of LineMOD dataset by reannotation. The dataset includes 8 objects in a cluttered scene. The main challenge of this dataset is very heavy occlusion, making this dataset very challenging. We fellow previous works [18] to only use this dataset for evaluation by a model trained on LineMOD dataset.

\subsection{Metrics}

In our experiments, we use two common metrics for evaluation: ADD and ADD-S. For asymmetric objects, the ADD metric is defined as the point-pair average distance between model points transformed by the predicted and the ground truth pose:

$$
A D D=\frac{1}{N} \sum_{x \in O}|(\hat{R} x+\hat{t})-(R x+t)|_{2}
$$

where $x$ denotes an object point $O, \hat{R}$ and $\hat{t}$ are predicted rotation and translation, $R$ and $t$ are ground truth rotation and translation. 
Table 1: Ablation studies of different novelties on Occlusion LineMOD dataset. The metric used is ADD(-S) accuracy. "G attention" means geometry attention mechanism. "A attention" means appearance attention mechanism.

\begin{tabular}{cccc}
\hline & Method & Accuracy \\
\hline G attention & A attention & \\
\hline$\times$ & $\times$ & 59.7 & 52.0 \\
& $\checkmark$ & $\checkmark$ & 50.6 \\
\\
$\times$ & $\checkmark$ & 52.4 & \\
\hline
\end{tabular}

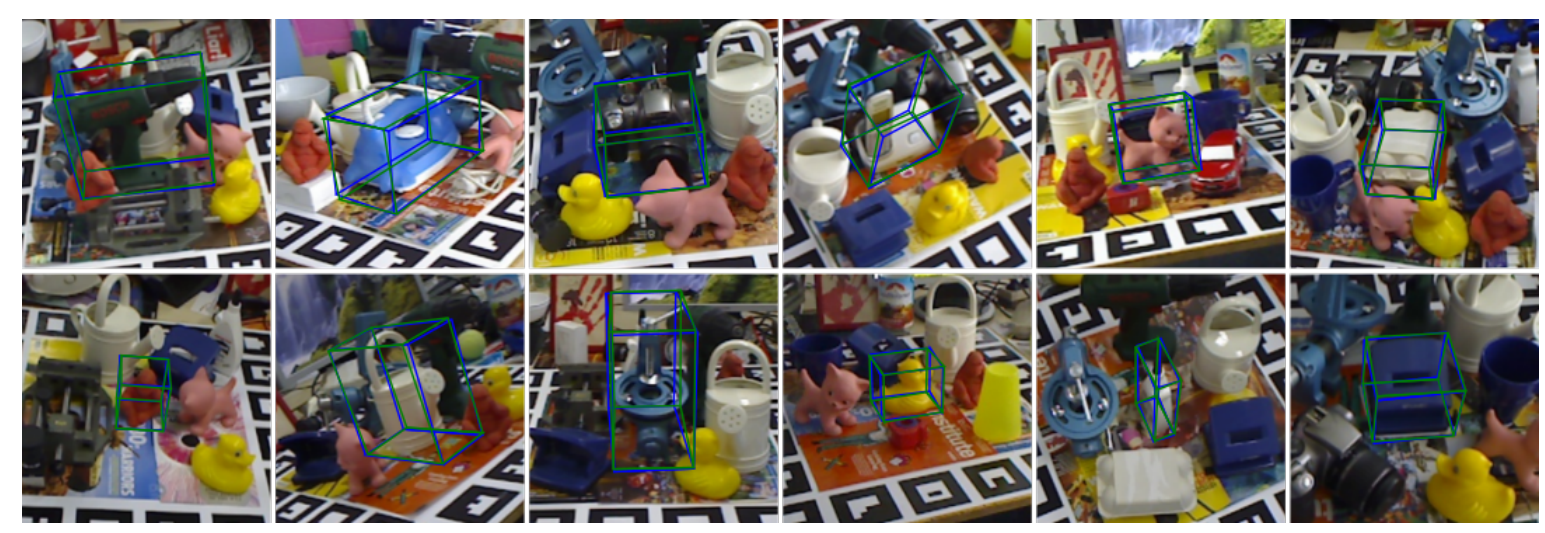

Figure 3: Visualization results on LineMOD dataset. The green and blue bounding boxes represent the ground truth pose and the estimated pose respectively.

For symmetric objects, the ADD-S metric calculates the distance between closest points:

$$
A D D-S=\frac{1}{N} \sum_{x_{2} \in O} \min _{x_{1} \in O}\left\|\left(\hat{R} x_{1}+\hat{t}\right)-\left(R x_{2}+t\right)\right\|_{2}
$$

The predicted $6 \mathrm{D}$ pose is considered to be correct if the average distance is less than $10 \%$ of the object model's diameter.

\subsection{Implementation details}

The graph neural network is constructed using $\mathrm{k}=16$ nearest neighbors. During training and inference, we randomly sampled 500 points for each object as input. The overall loss is $L=L_{R}+\lambda_{1} L_{\text {reg }}+$ $\lambda_{2} L_{t}$, and the balance factor in loss is set as $\lambda_{1}=3$ and $\lambda_{2}=6$ by empirical evaluation. We train the model for 50 epochs with a batch size of 8. We utilized RAdam [12] with Lookahead [27] as the optimizer. The initial learning rate is set to $5 \mathrm{e}-4$ and decay using a cosine schedule. For refinement, we use the colored ICP provided by Open3D and iterate 20 times.

\subsection{Ablation studies}

In this work, our proposed methods have two novelties. First, we extract the feature by graph neural network with an attention mechanism based on geometry and appearance. We compare the different attention mechanisms in Table 1 . We first use only the feature to calculate the weights and get an accuracy of $49.7 \%$. Then we add the geometry-based attention mechanism to the network, which can greatly improve the accuracy. The appearance-based attention mechanism can further improve network performance. This is mainly due to the full use of two streams of information.

\subsection{Results on the benchmark dataset}

We report our evaluation results of the pose estimation experiments on the LineMOD dataset (see Table 2). Our method achieves $98.7 \%$ accuracy without refinement which is outperforming the other methods. We have to clarify that all the results listed here use the same segmentation masks released by PoseCNN. Some visualizations of the results are present in Figure 3. It is worth noting that, our method can outperform those methods that require refinement without using any refinement. Especially, due to our attention mechanism, our improvement on objects with complex shapes and rich textures such as 'cam', 'holepuncher' and 'phone' is more obvious.

We use the model trained on the LineMOD for evaluation on Occlusion LineMOD, shown in Table 2. For the ADD(-s) metric, our method achieves the best performance. We achieved an accuracy of $52.4 \%$, which greatly exceeds other methods. This result shows that our network with the attention mechanism is robust to occlusion. We show some visualization results in Figure 4. It can be observed that our method is robust to hard cases with occlusion and the colored ICP can further improve performance.

\subsection{Running time}

Our pose estimation method infers at 0.08 s per instance on a laptop with an Intel i7 4.2GHz CPU and a single GTX $1080 \mathrm{GPU}$, which is fast enough for real-time pose estimation. 
Table 2: ADD(-S) performance on LineMOD dataset

\begin{tabular}{|c|c|c|c|c|c|c|}
\hline & \multicolumn{3}{|c|}{ RGB } & \multicolumn{3}{|c|}{ RGB-D } \\
\hline & $\begin{array}{c}\text { PoseCNN } \\
\text { DeepIM }[10,23]\end{array}$ & PVNet [18] & CDPN [11] & $\begin{array}{c}\text { Dense-Fusion } \\
\text { [22] }\end{array}$ & Tian et al. [21] & Ours \\
\hline ape & 77.0 & 43.6 & 64.4 & 92.3 & 85.0 & 96.2 \\
\hline benchvise & 97.5 & 99.9 & 97.8 & 93.2 & 95.5 & 98.0 \\
\hline cam & 93.5 & 86.9 & 91.7 & 94.4 & 91.3 & 99.4 \\
\hline can & 96.5 & 95.5 & 95.9 & 93.1 & 95.2 & 98.6 \\
\hline cat & 82.1 & 79.3 & 83.8 & 96.5 & 93.6 & 99.2 \\
\hline driller & 95.0 & 96.4 & 96.2 & 87.0 & 82.6 & 98.7 \\
\hline duck & 77.7 & 52.6 & 66.8 & 92.3 & 88.1 & 95.8 \\
\hline eggbox & 97.1 & 99.2 & 99.7 & 99.8 & 99.9 & 100.0 \\
\hline glue & 99.4 & 95.7 & 99.6 & 100.0 & 99.6 & 100.0 \\
\hline holepuncher & 52.8 & 81.9 & 71.8 & 86.9 & 92.6 & 99.3 \\
\hline iron & 98.3 & 98.9 & 97.9 & 97.0 & 95.9 & 99.0 \\
\hline lamp & 97.5 & 99.3 & 97.9 & 95.3 & 94.4 & 99.4 \\
\hline phone & 87.7 & 92.4 & 90.8 & 92.8 & 93.6 & 98.0 \\
\hline average & 88.6 & 86.3 & 89.9 & 93.3 & 92.9 & 98.6 \\
\hline
\end{tabular}
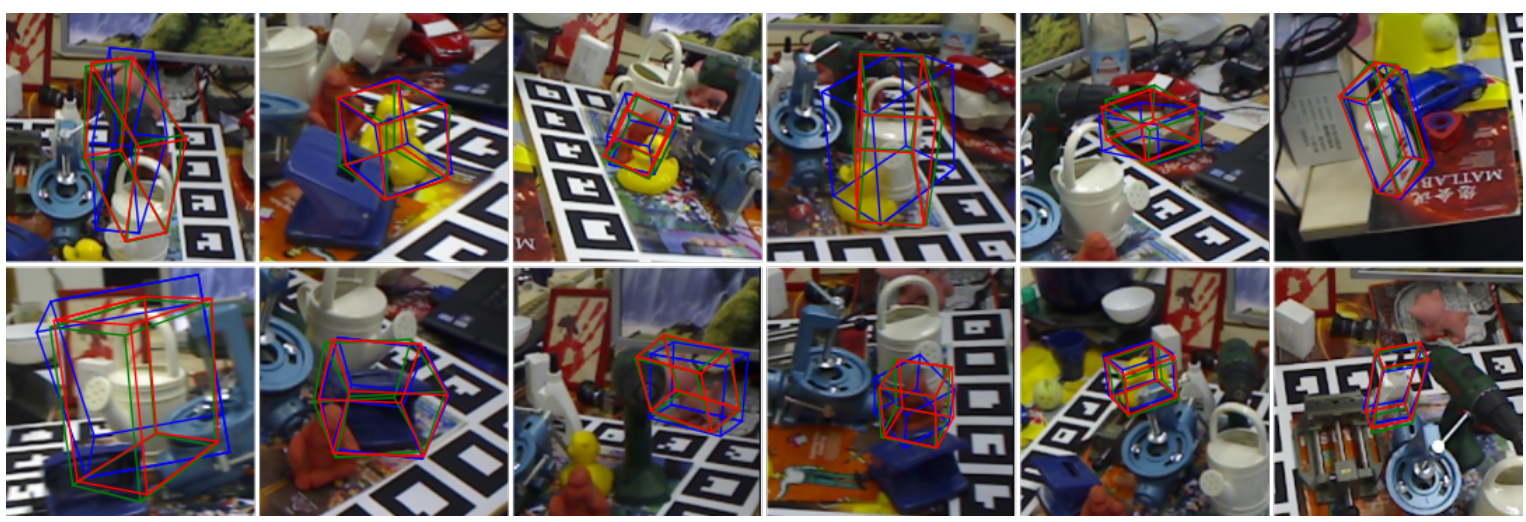

Figure 4: Visualization results on Occlusion LineMOD dataset. The green bounding box represents the ground truth pose, the blue bounding box is the initial predicted pose, and the red bounding box is the pose refined by colored ICP.

Table 3: ADD(-S) performance on Occlusion LineMOD dataset

\begin{tabular}{lccccc}
\hline & PVNet [18] & Pix2Pose [17] & DPOD [26] & DPVL [25] & Ours + Colored ICP \\
\hline ape & 15.8 & 22.0 & - & 19.2 & $\mathbf{6 9 . 8}$ \\
can & 63.3 & 44.7 & - & 21.1 & 41.9 \\
cat & 16.7 & 22.7 & - & $\mathbf{7 1 . 7}$ & $\mathbf{4 0 . 9}$ \\
driller & 65.7 & 44.7 & - & 34.3 & 50.7 \\
duck & 25.2 & 15.0 & - & 47.3 & $\mathbf{3 5 . 6}$ \\
eggbox & 50.2 & 25.2 & - & 39.7 & $\mathbf{7 8 . 1}$ \\
glue & 49.6 & 32.4 & - & 45.3 & 48.9 \\
holepuncher & 39.7 & $\mathbf{4 9 . 5}$ & 47.3 & 43.5 & $\mathbf{5 2 . 4}$ \\
average & 40.8 & 32.0 & & \\
\hline
\end{tabular}

\section{CONCLUSION}

We propose a novel attention based graph neural network for 6D object pose estimation from colored point clouds. The attention mechanism is based on geometric and appearance information. The rotation transformation is solved by a robust discrete-continuous regression scheme, while the translation transformation is by voting to the center. In hard cases, the final estimated pose is refined by 
colored ICP. Ablation studies show the effectiveness of our proposed attention mechanism. Experimental results on benchmark datasets show that our method can effectively utilize the spatial geometric and appearance information of objects, which is better than other methods in terms of accuracy and efficiency.

In future work, we would consider using self-supervision to extend our work to eliminate the dependence on labeled data. Another extension is to introduce it into the robot grasping algorithm.

\section{REFERENCES}

[1] Besl, P. J., \& McKay, N. D. (1992, April). Method for registration of 3-D shapes. In Sensor fusion IV: control paradigms and data structures (Vol. 1611, pp. 586-606). International Society for Optics and Photonics.

[2] Brachmann, E., Krull, A., Michel, F., Gumhold, S., Shotton, J., \& Rother, C. (2014, September). Learning $6 \mathrm{~d}$ object pose estimation using $3 \mathrm{~d}$ object coordinates. In European conference on computer vision (pp. 536-551). Springer, Cham.

[3] Do, T., Pham, T., Cai, M., \& Reid, I. (2019). Real-time monocular object instance $6 \mathrm{~d}$ pose estimation.

[4] Hinterstoisser, S., Holzer, S., Cagniart, C., Ilic, S., Konolige, K., Navab, N., \& Lepetit, V. (2011, November). Multimodal templates for real-time detection of texture-less objects in heavily cluttered scenes. In 2011 international conference on computer vision (pp. 858-865). IEEE.

[5] Hinterstoisser, S., Lepetit, V., Ilic, S., Holzer, S., Bradski, G., Konolige, K., \& Navab, N. (2012, November). Model based training, detection and pose estimation of texture-less $3 \mathrm{~d}$ objects in heavily cluttered scenes. In Asian conference on computer vision (pp. 548-562). Springer, Berlin, Heidelberg.

[6] Hu, Y., Hugonot, J., Fua, P., \& Salzmann, M. (2019). Segmentation-driven 6d object pose estimation. In Proceedings of the IEEE/CVF Conference on Computer Vision and Pattern Recognition (pp. 3385-3394).

[7] Kehl, W., Manhardt, F., Tombari, F., Ilic, S., \& Navab, N. (2017). Ssd-6d: Making rgb-based $3 \mathrm{~d}$ detection and $6 \mathrm{~d}$ pose estimation great again. In Proceedings of the IEEE international conference on computer vision (pp. 1521-1529).

[8] Labbé, Y., Carpentier, J., Aubry, M., \& Sivic, J. (2020, August). CosyPose: Consistent multi-view multi-object 6D pose estimation. In European Conference on Computer Vision (pp. 574-591). Springer, Cham.

[9] Li, C., Bai, J., \& Hager, G. D. (2018). A unified framework for multi-view multi-class object pose estimation. In Proceedings of the European Conference on Computer Vision (ECCV) (pp. 254-269)

[10] Li, Y., Wang, G., Ji, X., Xiang, Y., \& Fox, D. (2018). Deepim: Deep iterative matching for $6 \mathrm{~d}$ pose estimation. In Proceedings of the European Conference on Computer Vision (ECCV) (pp. 683-698).

[11] Li, Z., Wang, G., \& Ji, X. (2019). Cdpn: Coordinates-based disentangled pose network for real-time rgb-based 6-dof object pose estimation. In Proceedings of the IEEE/CVF International Conference on Computer Vision (pp. 7678-7687).

[12] Liu, L., Jiang, H., He, P., Chen, W., Liu, X., Gao, J., \& Han, J. (2019). On the variance of the adaptive learning rate and beyond. arXiv preprint arXiv:1908.03265.

[13] Lowe, D. G. (1999, September). Object recognition from local scale-invariant features. In Proceedings of the seventh IEEE international conference on computer vision (Vol. 2, pp. 1150-1157). Ieee.

[14] Manhardt, F., Kehl, W., Navab, N., \& Tombari, F. (2018). Deep model-based 6d pose refinement in rgb. In Proceedings of the European Conference on Computer Vision (ECCV) (pp. 800-815).

[15] Michel, F., Kirillov, A., Brachmann, E., Krull, A., Gumhold, S., Savchynskyy, B., \& Rother, C. (2017). Global hypothesis generation for 6D object pose estimation. In Proceedings of the IEEE Conference on Computer Vision and Pattern Recognition (pp. 462-471).

[16] Park, J., Zhou, Q. Y., \& Koltun, V. (2017). Colored point cloud registration revisited. In Proceedings of the IEEE international conference on computer vision (pp. 143-152).

[17] Park, K., Patten, T., \& Vincze, M. (2019). Pix2pose: Pixel-wise coordinate regression of objects for $6 \mathrm{~d}$ pose estimation. In Proceedings of the IEEE/CVF International Conference on Computer Vision (pp. 7668-7677).

[18] Peng, S., Liu, Y., Huang, Q., Zhou, X., \& Bao, H. (2019). Pvnet: Pixel-wise voting network for 6 dof pose estimation. In Proceedings of the IEEE/CVF Conference on Computer Vision and Pattern Recognition (pp. 4561-4570).

[19] Rios-Cabrera, R., \& Tuytelaars, T. (2013). Discriminatively trained templates for $3 \mathrm{~d}$ object detection: A real time scalable approach. In Proceedings of the IEEE international conference on computer vision (pp. 2048-2055).

[20] Tekin, B., Sinha, S. N., \& Fua, P. (2018). Real-time seamless single shot 6 d object pose prediction. In Proceedings of the IEEE Conference on Computer Vision and Pattern Recognition (pp. 292-301).

[21] Tian, M., Pan, L., Ang, M. H., \& Lee, G. H. (2020, May). Robust 6d object pose estimation by learning rgb-d features. In 2020 IEEE International Conference on Robotics and Automation (ICRA) (pp. 6218-6224). IEEE.

[22] Wang, C., Xu, D., Zhu, Y., Martín-Martín, R., Lu, C., Fei-Fei, L., \& Savarese, S. (2019). Densefusion: 6d object pose estimation by iterative dense fusion. In Proceedings of the IEEE/CVF Conference on Computer Vision and Pattern Recognition (pp. 33433352).

[23] Xiang, Y., Schmidt, T., Narayanan, V., \& Fox, D. (2017). Posecnn: A convolutional neural network for $6 \mathrm{~d}$ object pose estimation in cluttered scenes. arXiv preprint arXiv:1711.00199.

[24] Yan, Y., \& Chirikjian, G. S. (2012, May). Almost-uniform sampling of rotations for conformational searches in robotics and structural biology. In 2012 IEEE International Conference on Robotics and Automation (pp. 4254-4259). IEEE.

[25] Yu, X., Zhuang, Z., Koniusz, P., \& Li, H. (2020). 6dof object pose estimation via differentiable proxy voting loss. arXiv preprint arXiv:2002.03923.

[26] Zakharov, S., Shugurov, I., \& Ilic, S. (2019). Dpod: 6d pose object detector and refiner. In Proceedings of the IEEE/CVF International Conference on Computer Vision (pp. 1941-1950).

[27] Zhang, M. R., Lucas, J., Hinton, G., \& Ba, J. (2019). Lookahead optimizer: k steps forward, 1 step back. arXiv preprint arXiv:1907.08610. 